\title{
Contact analysis and surface optimization of crowning gear coupling
}

\author{
J. Wei, Y. Yao \& K. Zhang \\ Traction Power State Key Laboratory, \\ Southwest Jiaotong University, China
}

\begin{abstract}
In order to ensure that coupling works smoothly, securely and effectively in conditions of required misalignment, the gear geometry is designed carefully and the contact performances of the crowned gear coupling is improved.

The clearances between teeth were calculated through the geometry method and the position of minimum clearance is recognized as the contacted point. The clearance result at a certain misalignment angle has been confirmed by Finite Element Analysis. It is clear that the position of contact point is influenced by the misalignment and the crowning radius of hub teeth.

Taking the teeth bending deformation into account under load, the distribution law of teeth surface force can be worked out according to the clearance and the stiffness of each tooth can be estimated through a taper cantilever beam model. The result shows that the maximum of teeth load increases with the misalignment and the crowning radius.

The study optimizes the parameters of three sections arc surface for the hub teeth according to the mechanical analysis of coupling, in order to reduce the contact stress on teeth and meet the requirements of the working misalignment. The radius of the flank curve should be shorter than that of the middle arc, so that the requirements of smooth contact were met in cases of a large misalignment angle and large contact curvature was avoided in normal conditions.
\end{abstract}

Keywords: crowning gear coupling, misalignment angle, clearance between meshing teeth. 


\section{Introduction}

Crowning gear coupling is a kind of moveable rigid coupling which is widely used in the mechanical domain. It transmits the torque from the input axis to the output one and fits the misalignment between connected elements. Recently, the high speed train boom in China and the CRH series has come into service heavily. Crowning gear coupling is used on the railways very frequently, so that it becomes more important to study crowning gear coupling comprehensively in order to improve the performance of it.

Crowning gear coupling mainly consists of the hub which is connected with the input or output axis and the sleeve which is meshed with hubs. The crowning surface applies to hub teeth only and the sleeve uses straight teeth with modification. The modification can causes gaps between meshing teeth which generate the space for lubrication and misalignment. Generally speaking, the sleeve modifies the teeth tangentially changing the teeth thickness only, which is different to radial modification.

Crowning gear coupling is developed from straight teeth gear coupling which requires the input and output axis to be accurately coaxial and causes the edge contact easily. Compared with straight teeth, crowning teeth can protect the teeth surface from harmful contact in cases where the coupling is running with a misalignment, and improves the wear life of the coupling (as verified by Mancuso and Ziberman [1]).

According to a study of the related documents, there have been studies on many styles of crowning gear coupling. As early as 1968, Moked's [2] study analysed the gear coupling by the method of mathematics, which is mainly about the contact characteristics, the load distribution on each tooth and so on. Then Alfares et al. [3] studied the clearance between meshing teeth by a method of geometry. The calculation reflected the geometrical state between the meshing teeth and analysed the effect of the key parameters, such as the pressure angle, module and the crowning, on the performance of the coupling. Recently, Kelemen [4] analysed the load carrying capacity of gear coupling by the Hertz theory method.

This study is based on the calculation of the clearance between meshing teeth, which makes use of an improved geometry method which reflects the crowning surface more accurately according to the method of manufacture.

\section{Clearance between meshing teeth}

\subsection{Calculation method}

The coordinate systems shown in fig. 1 are:

$\mathrm{S} 1$ (o, x1, y1, z1), S10 (o, x10, y10, z10)

S2 (o, x2, y2, z2), S20 (o, x20, y20, z20) 


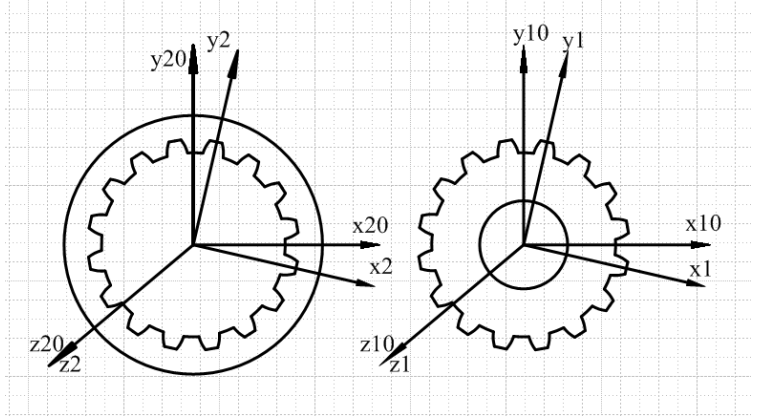

Figure 1: Coordinate system.

According to the principle of manufacture of the crowning gear coupling, which is shown in fig. 2, the hobbing cutter translates along a curve and the surface of the teeth is formed by the rotation of the involute line A in fig. 3 .

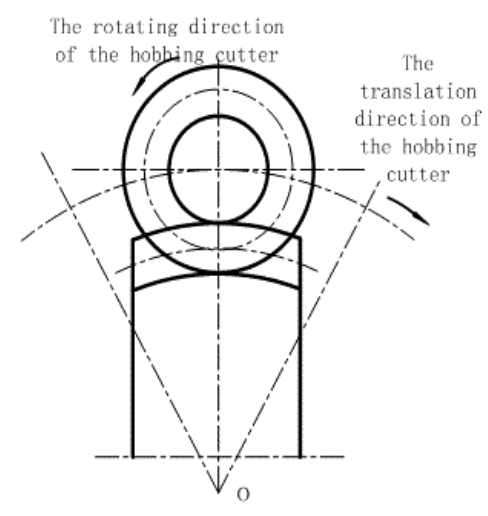

Figure 2: The manufacture of the hub.

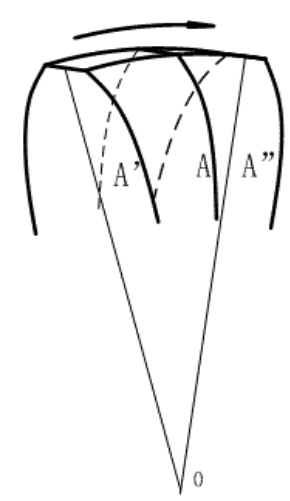

Figure 3: The rotation of the involution.

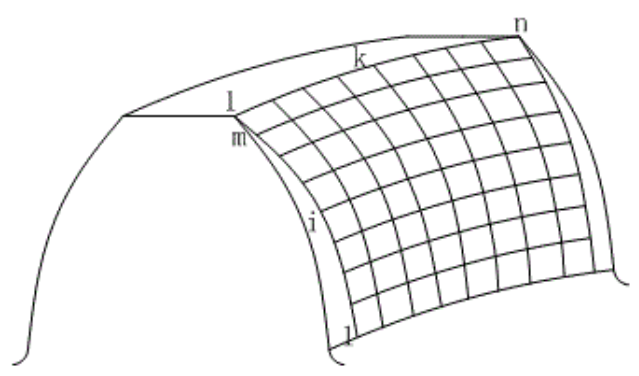

Figure 4: The meshed teeth surface. 
The teeth surface is meshed as fig. 4 . The node $(\mathrm{i}, \mathrm{k})$ is a node rotated from the node $(\mathrm{i}, \mathrm{n} / 2+1)$, so that

$$
y_{h k i}^{(1)}=y_{h k_{m i} i}^{(1)}
$$

where

$$
k_{m}=(n+1) / 2 .
$$

In the coordinate system $S_{l}$

$$
y_{h k_{m i} i}^{(1)}=r_{h i} \sin \left(\beta_{h i} / 2\right)
$$

According to the involute characteristics, $\beta_{h i}$ is the tooth thickness angle at the position of node $\left(\mathrm{i}, \mathrm{k}_{\mathrm{m}}\right)$, which can be calculated by

$$
\beta_{h i}=\frac{T_{h p}}{r_{h p}}+2\left(\operatorname{inv}\left(\alpha_{h p}\right)-i n v\left(\alpha_{h i}\right)\right)
$$

where

$T_{h p}$ is the tooth thickness of pitch circle of the hub.

$r_{h p}$ is the radius of pitch circle of the hub.

$r_{h i}$ is the distance from the node $\left(\mathrm{i}, \mathrm{k}_{\mathrm{m}}\right)$ to the axis of the hub.

$\alpha_{\text {hp }}$ is the pressure angle of pitch circle.

$\alpha_{\mathrm{hi}}$ is the pressure angle at node, which is calculated by $\alpha_{h i}=\cos ^{-1}\left(r_{p} \cos \left(\alpha_{h p}\right) / r_{h i}\right)$.

The coordinate of node $(\mathrm{i}, \mathrm{k})$ in coordinate system $S_{l}$ is obtained by

$$
\begin{aligned}
& y_{h k i}^{(1)}=y_{h k_{m} i}^{(1)} \\
& x_{h k i}^{(1)}=\left(r_{c i}-r_{h i}\left(1-\cos \left(\beta_{h i} / 2\right)\right)\right) \cos \left(\alpha_{c k}\right)-\left(r_{c i}-r_{h i} \cos \left(\beta_{h i} / 2\right)\right. \\
& z_{h k i}^{(1)}=\sin \left(\alpha_{c k}\right) r_{c i}
\end{aligned}
$$

where

$r_{c i}$ is the distance from the node $(\mathrm{i}, \mathrm{k})$ to the center of the translate curve of the hobbing cutter,

$\alpha_{\mathrm{ck}}$ is the rotation angle from the node $\left(\mathrm{i}, \mathrm{k}_{\mathrm{m}}\right)$ to the node (i,k) cross the center of the translate curve of the hobbing cutter.

The coordinate of node (i,k) in coordinate system $S_{20}$ is obtained by

$$
\left[\begin{array}{l}
x_{h k i}^{(20)} \\
y_{h k i}^{(20)} \\
z_{h k i}^{(20)}
\end{array}\right]=M_{20,10} M_{10,1}\left[\begin{array}{l}
x_{h k i}^{(1)} \\
y_{h k i}^{(1)} \\
z_{h k i}^{(1)}
\end{array}\right]
$$

where $M_{20,10}$ is the rotational matrix between $S_{10}$ and $S_{20}$, and $M_{10,1}$ is the rotational matrix between $S_{10}$ and $S_{I}$.

$$
M_{20,10}=\left[\begin{array}{ccc}
1 & 0 & 0 \\
0 & \cos (\theta) & -\sin (\theta) \\
0 & \sin (\theta) & \cos (\theta)
\end{array}\right], M_{10,1}=\left[\begin{array}{ccc}
\cos \left(\varphi_{h}\right) & -\sin \left(\varphi_{h}\right) & 0 \\
\sin \left(\varphi_{h}\right) & \cos \left(\varphi_{h}\right) & 0 \\
0 & 0 & 1
\end{array}\right]
$$

$\theta$ is the misalignment angle of gear coupling, $\varphi_{h}$ is the rotation angle of the hub. 
The node on the sleeve which may mesh with the node $(\mathrm{k}, \mathrm{i})$ is at the position of $\left(x_{s k i}^{(2)}, y_{s k i}^{(2)}, z_{s k i}^{(2)}\right)$.

$$
\begin{aligned}
& x_{s k i}^{(2)}=r_{s k i} \cos \left(\beta_{s k i} / 2\right) \\
& y_{h k i}^{(2)}=r_{s k i} \sin \left(\beta_{s k i} / 2\right)
\end{aligned}
$$

where

$r_{s k i}$ is the distance from the point $\left(x_{s k i}^{(2)}, y_{s k i}^{(2)}, z_{s k i}^{(2)}\right)$ to the axis of the sleeve,

$\beta_{s k i}$ is the tooth thickness the angle at the position $\left(x_{s k i}^{(2)}, y_{s k i}^{(2)}, z_{s k i}^{(2)}\right)$, which can be calculated by

$$
\beta_{s k i}=\frac{T_{s p}}{r_{s p}}+2\left(i n v\left(\alpha_{s p}\right)-i n v\left(\alpha_{s k i}\right)\right)
$$

where

$T_{s p}$ is the tooth thickness of the pitch circle of the sleeve,

$r_{s p}$ is the radius of the pitch circle of the sleeve,

$r_{s k i}$ is the distance from the point $\left(x_{s k i}^{(2)}, y_{s k i}^{(2)}, z_{s k i}^{(2)}\right)$ to the axis of the sleeve,

which is obtained by $r_{s k i}=\sqrt{\left(x_{h k i}^{(20)}\right)^{2}+\left(y_{h k i}^{(20)}\right)^{2}}$.

$\alpha_{\mathrm{sp}}$ is the pressure angle of pitch circle of the sleeve,

$\alpha_{\text {ski }}$ is the pressure angle at point $\left(x_{s k i}^{(2)}, y_{s k i}^{(2)}, z_{s k i}^{(2)}\right)$.

The coordinate of point $\left(x_{s k i}^{(2)}, y_{s k i}^{(2)}, z_{s k i}^{(2)}\right)$ in coordinate system $S_{20}$ is obtained by

$$
\left[\begin{array}{c}
x_{s k i}^{(20)} \\
y_{s k i}^{(20)} \\
z_{s k i}^{(20)}
\end{array}\right]=M_{20,2}\left[\begin{array}{c}
x_{s k i}^{(2)} \\
y_{s k i}^{(2)} \\
z_{s k i}^{(2)}
\end{array}\right]
$$

where $M_{20,2}$ is the rotational matrix between $S_{2}$ and $S_{20}$.

$$
M_{20,2}=\left[\begin{array}{ccc}
\cos \left(\varphi_{s}\right) & -\sin \left(\varphi_{s}\right) & 0 \\
\sin \left(\varphi_{s}\right) & \cos \left(\varphi_{s}\right) & 0 \\
0 & 0 & 1
\end{array}\right]
$$

The clearance between the node $(\mathrm{i}, \mathrm{k})$ and the point $\left(x_{s k i}^{(20)}, y_{s k i}^{(20)}, z_{s k i}^{(20)}\right)$ is obtained by

$$
\text { clearance }=r_{s k i}\left(\tan ^{-1}\left(\frac{y_{s k i}^{(20)}}{x_{s k i}^{(20)}}\right)-\tan ^{-1}\left(\frac{y_{h k i}^{(20)}}{x_{h k i}^{(20)}}\right)\right)
$$

\subsection{Result and analysis}

Fig. 5 shows the distribution of the minimum clearance between the meshed teeth. The minimum clearance changes with the angle of teeth. The clearance in the flip area is smaller than that of the other teeth, which means that the teeth in the flip area mesh more easily when the coupling is running with a misalignment angle. 
Fig. 6 shows the clearance of the teeth when the misalignment angle is $0.7^{\circ}$. The node with the minimum clearance is judged as the contact point, the position of which changes with the rotation of coupling. The contact point tends to concentrate on the tip of the tooth when the hub rotates from $0^{\circ}$ to $90^{\circ}$.

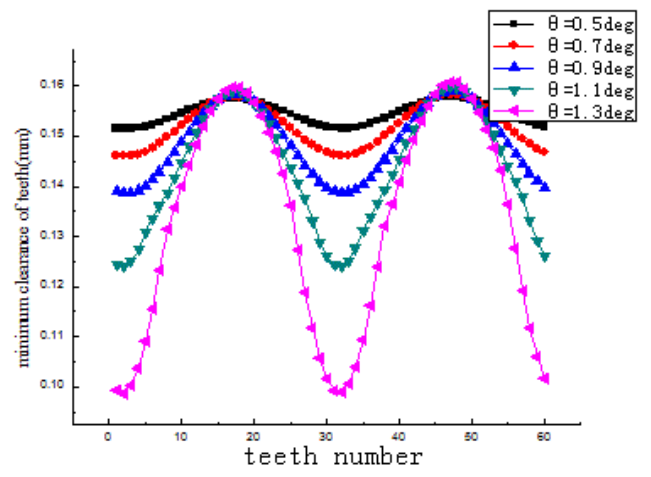

Figure 5: Clearance of each tooth.
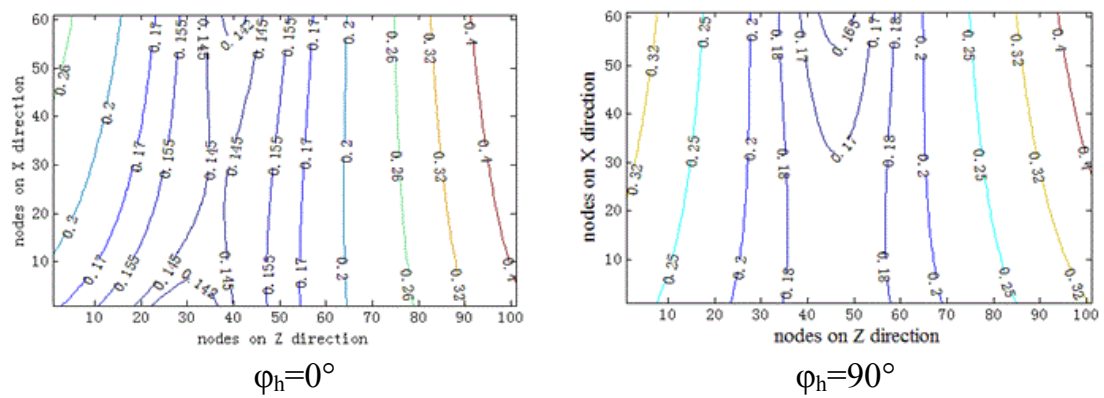

Figure 6: Clearance distribution on the teeth at $\theta=0.7^{\circ}$.

Fig. 7 shows the distribution of the clearance between teeth of the flip area in the condition of a different misalignment angle. The minimum clearance on the teeth occurs at the tip of the teeth when the misalignment angle is smaller than $0.5^{\circ}$, distributes at both the root and the tip of the teeth when the misalignment angle increases and concentrates on the root of the teeth when the misalignment angle exceeds $0.9^{\circ}$. This trend can be proved by the result of the method of FEM shown in fig. 8.

Taking the elasticity of the contacted surface into account, the teeth contact at two points, which generate the ' $\mathrm{X}$ ' spot on the teeth, when the extreme value of clearance occurs at both the tip and the root of the teeth. 

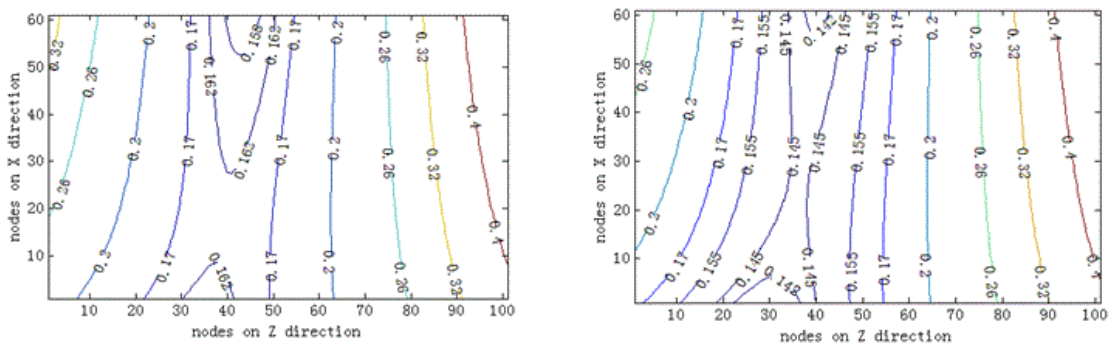

$\theta=0.5^{\circ}$

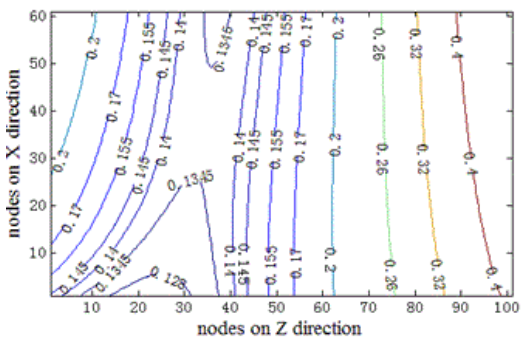

$\theta=0.9^{\circ}$ $\theta=0.7^{\circ}$

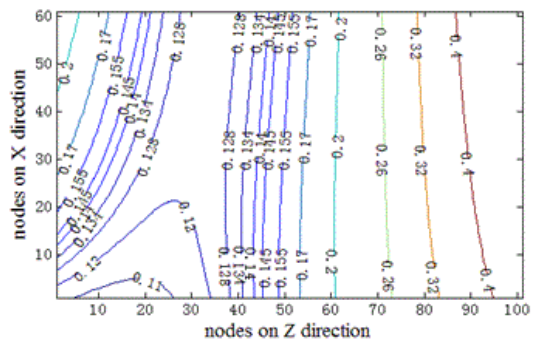

$\theta=1.1^{\circ}$

Figure 7: Clearance distribution on the teeth of the flip area.

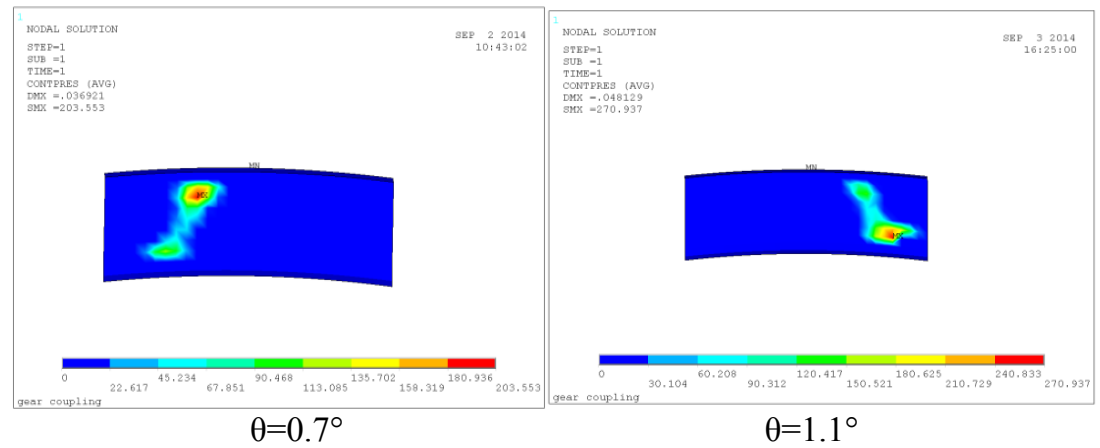

Figure 8: Contact stress distribution obtained by FEM.

Fig. 9 shows the contact point changing with the rotation of the hub on the direction of tooth width in conditions of different misalignment angle. The contact point deviates from the center line of the teeth surface when the misalignment angle increases, and the maximum of the deviation occurs at the flip area. The position of the contact point blinks at some angle when the misalignment is greater than $0.5^{\circ}$, which is because the contact point changes from the top to the bottom of the teeth surface. 


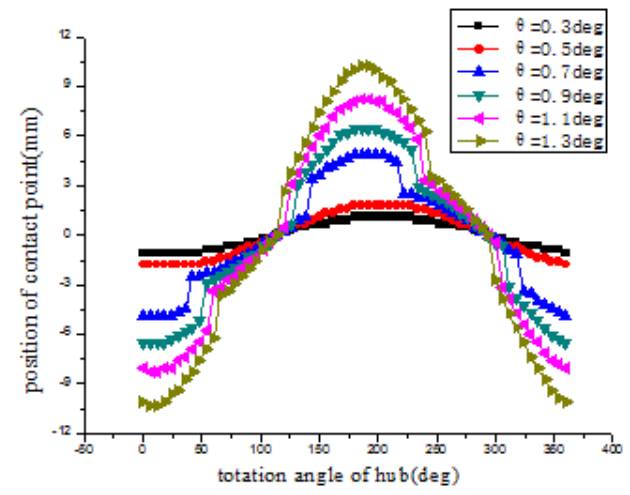

Figure 9: The position of contact point.

\section{Distribution law of teeth surface force}

Taking the bending deformation of the teeth into account, the distribution of the load on teeth follows the law of the stiffness. Calculating the bending stiffness of teeth by a taper cantilever beam model is shown in fig. 10 .

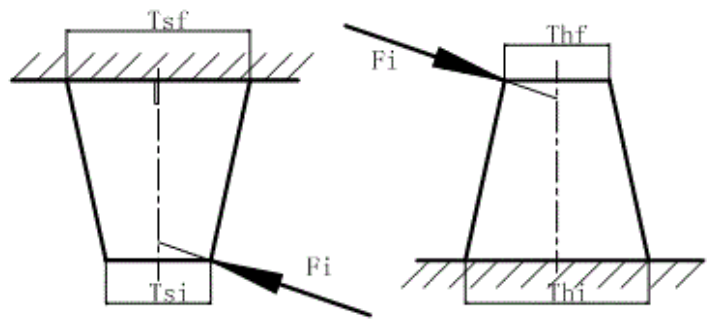

Figure 10: Taper cantilever beam model of teeth.

The load on teeth surface distribute with the sum deflection of the pairs of meshed teeth. According to the minimum clearance of every tooth and the torque upon the coupling, the distribution of load on teeth is worked out.

Fig. 11 shows the distribution of the load on each tooth surface in the case of a different misalignment angle. The maximum load occurs in the flip area where the clearance between teeth is small. Fig. 12 shows that the maximum teeth load increases with the misalignment when the coupling runs with a different torque. The maximum teeth load is almost the same when the misalignment angle is smaller than $0.5^{\circ}$ because all the teeth become meshed at nearly the same position. When the misalignment angle is over $0.7^{\circ}$ some of the contact points transfer from the tip to the root of the teeth and the teeth at the swing area gradually unmesh so that the maximum load on teeth increases with the misalignment significantly. 


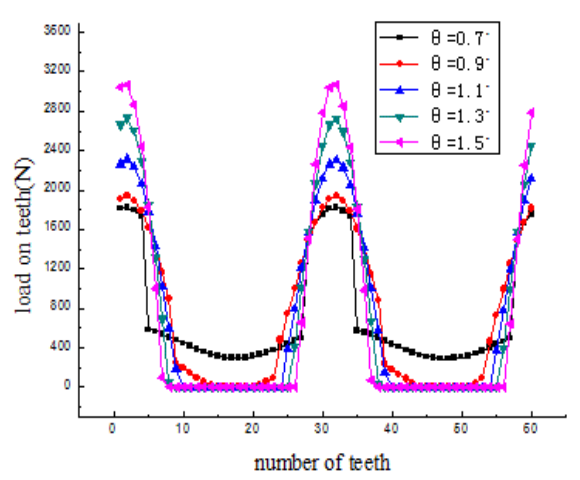

Figure 11: Distribution of the teeth load.

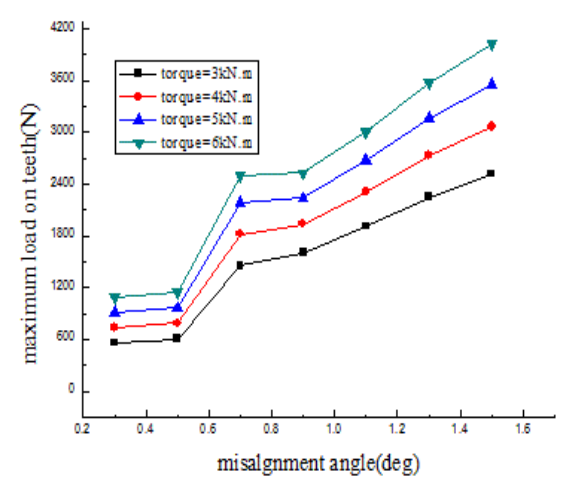

Figure 12: Maximum of the teeth load.

Because of the effect of the deviation of contact points and the friction between the meshed teeth, an additional moment occurs along a direction which is orthogonal to the axis of coupling.

Fig. 13 shows that the additional moment increases with the misalignment angle and the slope grows little by little as the misalignment is increased.

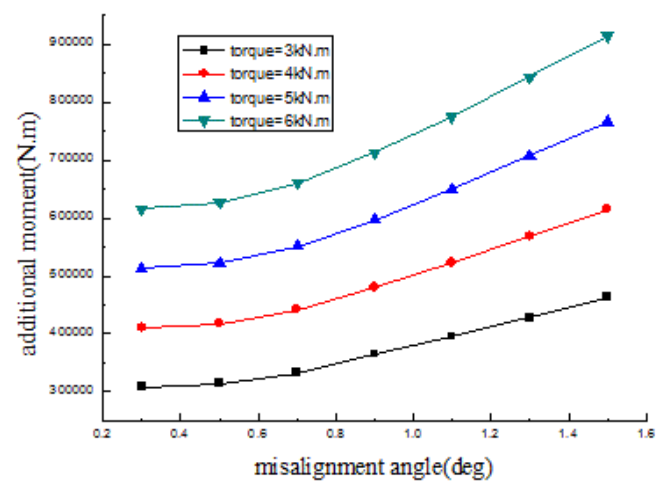

Figure 13: Additional moment of coupling.

\section{Optimization of the teeth surface parameters}

The contact point transfers from the middle to the seamed edge of the teeth when the misalignment angle increases according to the conclusion above, which may harm the teeth surface seriously. In order to protect the contact surface from the meshing at the seamed edge, the hub has to be carefully designed.

The critical misalignment, which can be used to distinguish the contact conditions, is defined as the maximum of the misalignment which will not cause 
the contact at the seamed edge. If the critical misalignment meets the requirement, the teeth mesh on the crowning surface securely.

Fig. 14 shows that the critical misalignment, $\theta_{c}$, is closely related to the press angle, the tip chamfering on the sleeve teeth and the crowning radius. The $\theta_{c}$ decreases rapidly as the crowning radius is shorter than $60 \mathrm{~mm}$, and it goes on decreasing with the slower and slower increase of the radius. The chamfering on the tip of the sleeve teeth and a larger press angle contribute to improving the capacity for the misalignment angle.

Fig. 15 shows the clearance between the teeth on the root section. The conjugate modification is taken at both sides of the teeth to eliminate the negative clearance in cases of extraordinary misalignment angle of $5^{\circ}$. Meanwhile, the middle curve of the crowning contact when the misalignment angle is small. This kind of modified crowning teeth is fit for the coupling that runs with a small misalignment in general, and needs to meet a specific large misalignment occasionally.

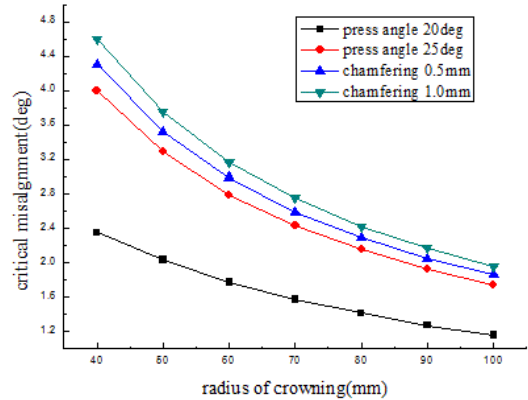

Figure 14: Critical misalignment angle.

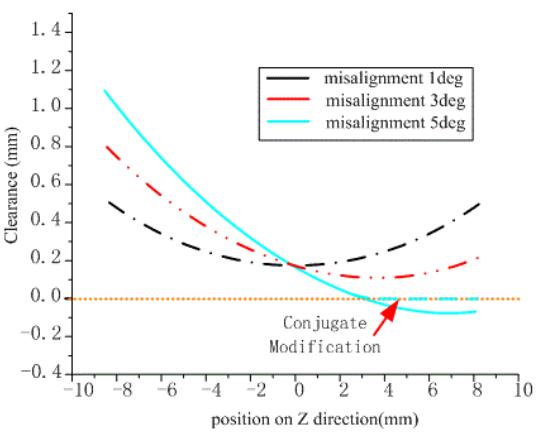

Figure 15: Curve of root teeth crowning.

\section{Conclusion}

The study reflects the meshing conditions of crowning gear coupling and obtains some conclusions by the calculation of clearance and the load on the teeth surface.

1. The minimum clearance changes with the rotation angle of coupling as a sine wave, and the amplitude which occurs at the flip area increases with the misalignment angle.

2. The contact point trend to concentrate on the top of the tooth when the hub rotates from $0^{\circ}$ to $90^{\circ}$ as the misalignment angle is $0.7^{\circ}$. The contact point at the flip area changes from the tip to the root of the teeth and deviates away from the center line of teeth surface along the axis direction as the misalignment angle increases.

3. The load on teeth is affected by the rotation angle of the teeth and reaches maximum at the flip area. The maximum load is almost the same with a 
small misalignment angle and increases with it as the misalignment is increased.

4. An additional moment is generated by the friction and the teeth load as the contact point deviates from the center line of the teeth. The additional moment increases with the misalignment angle and the slope becomes steeper when the misalignment angle increases.

5. The critical misalignment is closely related to the press angle, the tip chamfering on sleeve teeth and the crowning radius.

6. Conjugate modification can meet the requirements of a large misalignment angle in special conditions and contact at the middle curve of the crowning meanwhile.

\section{References}

[1] Jon R. Mancuso, Joe Ziberman, The Evolution of Gear Spindles. 2000 ASME Design Engineering Technical Conference, pp. 1-8, 2000.

[2] I. Moked, Tooth couplings - analysis and optimization. Journal of Engineering for Industry Transaction of ASME, Series B, 90(83), pp. 425434, 1968.

[3] M. A. Alfares, A. H. Falah, A. H. Elkholy, Clearance distribution of misaligned gear coupling teeth considering crowning and geometry variations. Mechanism and Machine Theory, 41, pp. 1258-1272, 2006.

[4] Laszlo Kekemen, Calculation of the load capacity of gear coupling based on the contact stress. Annals of Faculty Engineering Hunedoara, 2014. 